\title{
SISTEMAS DE EVALUACIÓN Y METODOLOGÍAS DOCENTES Y SU INCIDENCIA EN LAS COMPETENCIAS GENÉRICAS (EMICOG - PROFESORES)
}

\author{
Judit García-Martín \\ Universidad de Salamanca \\ jgarm@unileon.es \\ Jesús N. García \\ Universidad de León/Universidad de la Costa \\ Alicia J. Inciarte \\ Universidad de la Costa (Colombia) \\ Freddy V. Marín \\ Universidad de la Costa (Colombia) \\ Edgardo R. Sánchez \\ Universidad de la Costa (Colombia) \\ Marcial E. Conde \\ Universidad de la Costa (Colombia)
}

Fecha de Recepción: 7 Marzo 2019

Fecha de Admisión: 30 Abril 2019

\section{RESUMEN}

La proliferación de las herramientas y aplicaciones web sincrónicas, los sistemas de gestión de contenido, los cursos de enseñanza masivos y abiertos y los cambios constantes propios de la denominada sociedad de la información (SI), han conllevado que el principal reto del personal docente universitario sea su capacidad de adaptación e innovación. En este sentido, en la presente comunicación se describe el diseño de un instrumento ad hoc online, EMICOG - versión docente, diseñado a través de la herramienta SurveyMonkey. Con él, se pretenden estudiar las metodologías docentes aplicadas en las diversas asignaturas que imparte el profesorado de la Universidad de la Costa (Colombia), las formas de valoración o evaluación de las actividades transversales efectuadas así como sus prácticas docentes a través del análisis de seis secciones: i) datos de carácter general, ii) técnicas y estrategias instruccionales, iii) recursos utilizados en la evaluación y enseñanza de la asignatura, iv) variables psicoeducativas, v) necesidades formativas, y vi) procesos de enseñanza. Ha sido aplicado a 182 profesores universitarios, con el propósito de estudiar en qué medida estas actuaciones docentes pueden incidir en mejores resultados de competencias genéricas en las pruebas nacionales Saber Pro y en las variables psicoeducativas de los discentes, convirtiéndose 
pues en un instrumento de potenciación y mejora de la calidad docente y por consiguiente de los resultados académicos, mediante el esclarecimiento y la delimitación de las necesidades formativas, que serán la base de futuros programas/cursos instruccionales de estrategias eficientes para la mejora de la enseñanza y que repercutirán en la obtención de mayores logros académicos. Se trata de un Proyecto de Investigación Competitivo CONV-ÍNDEX Núm. 13-2018, Código Proyecto INV. 150-01-007-13 Universidad de la Costa (Colombia) - Universidad de León (España). Evaluado por la ACAC (MEN Colombia).

Palabras clave: sociedad de la información; universidad; instrumento; metodologías docentes

\section{ABSTRACT}

Assessment systems and teaching methodologies and their incidence in generic competences (EMICOG - TEACHERS). The proliferation of synchronous web tools and applications, learning management systems, massive open online courses and constant changes characteristic of the so-called information society (IS), have meant that the main challenge for university teaching staff be its capacity for adaptation and innovation. In this sense, the present communication describes the design of an online ad hoc instrument, EMICOG - teacher version, designed through the SurveyMonkey tool. With it, we intend to study the teaching methodologies applied in the various subjects taught by the teachers of the Universidad de la Costa (Colombia), the forms of assessment or evaluation of the transversal activities carried out as well as their teaching practices through the analysis of six sections: i) general data, ii) instructional strategies and techniques, iii) resources used in the evaluation and teaching of the subject, iv) psychoeducational variables, v) training needs, and vi) teaching processes. It has been applied to 182 university teachers, with the purpose of studying to what extent these teaching actions can affect better results of generic competences in the national tests Saber Pro and in the role of psychoeducational variables of the students, thus becoming an instrument of empowerment and improvement of the teaching quality and academic results, through the clarification and the delimitation of training needs, which will be the basis of future instructional programs / courses of efficient strategies for the improvement of teaching that will impact on obtaining higher academic achievements. It is a Competitive Research Project CONV-ÍNDEX Num. 13-2018, INV Project Code. 150-01-007-13 Universidad de la Costa (Colombia) - University of León (Spain). Evaluated by the ACAC (MEN Colombia).

Keywords: information society; university; instrument; teaching methodologies

\section{INTRODUCCIÓN}

En la actualidad, la capacidad de adaptación e innovación es un gran reto para el profesorado universitario (García-Martín y García-Sánchez, 2017) debido en la mayor parte de los casos, a la proliferación de las herramientas y aplicaciones web sincrónicas (García-Martín y García-Sánchez, 2013; García-Martín y García-Sánchez, 2015), los sistemas de gestión de contenido (García-Martín y García-Sánchez, 2018), Ios entornos virtuales de aprendizaje (Liaw y Huang, 2013; Lin, 2012), los cursos de enseñanza masivos y abiertos (García-Martín y García-Sánchez, 2019; García-Martín y García-Martín, 2018; Hew y Cheung, 2014) y los cambios constantes propios de la denominada sociedad de la información.

Todo ello, ha contribuido a revolucionar los procesos de enseñanza-aprendizaje creando la necesidad de que los profesores universitarios enseñen digitalmente mediante el uso de herramientas web diversas, la implementación de nuevas metodologías docentes, la adquisición y el desarrollo de competencias así como el uso de estrategias, estilos y enfoques de enseñanza innovadores (González, y Triviño, 2018). Por consiguiente, en este contexto, la capacitación en competencias 
genéricas, se convierte en el eje vertebrador de la formación permanente del profesorado y en la pieza angular de cualquier situación educativa (García-Martín y García-Sánchez, 2017).

Teniendo en cuenta estos antecedentes, en esta comunicación se describe el diseño de un instrumento ad hoc online, EMICOG - versión docente con el que se pretende estudiar las metodologías educativas aplicadas en las diversas asignaturas que imparte el profesorado participante, las formas de valoración o evaluación de las actividades transversales efectuadas así como sus prácticas docentes. Todo ello, con el fin de esclarecer y delimitar las necesidades formativas, que serán la base de futuros programas/cursos instruccionales de estrategias eficientes para la mejora de la enseñanza, repercutiendo en la consecución de mayores logros académicos. Igualmente, se incluyen escalas psicoeducativas de autoeficacia, de motivación, de inteligencia emocional, de estrategias de afrontamiento... a la par que se evalúan las estrategias que implementan de forma transversal en lectura crítica y construcción textual, así como los géneros textuales utilizados o los medios predominantes.

\section{PARTICIPANTES}

Durante el mes de abril, 249 profesores de la Universidad de la Costa (Colombia) decidieron participar voluntariamente en la investigación en curso. Si bien, es cierto, del profesorado inicial, solamente 182 ( $N=182,113$ hombres y 69 mujeres), con edades comprendidas entre los 24 y los 73 años de edad, respondieron a la práctica totalidad del cuestionario ad hoc, EMICOG en su versión docente (véase tabla 1).

Tabla 1

Distribución de los participantes por género y categoría profesional

\begin{tabular}{|c|c|c|c|c|c|}
\hline $\begin{array}{c}\text { Género/ } \\
\text { Categoría }\end{array}$ & Catedrático & Asistente & Adjunto & Titular & $\begin{array}{l}\text { Total del } \\
\text { Género }\end{array}$ \\
\hline Hombres & 17 & 69 & 17 & 10 & 113 \\
\hline $\begin{array}{c}\text { Edad } H \\
\text { M } \\
\text { (DT) }\end{array}$ & $\begin{array}{l}46 \text { años } \\
(13.70)\end{array}$ & $\begin{array}{l}39 \text { años } \\
(11.69)\end{array}$ & $\begin{array}{c}47 \text { años } \\
(9.36)\end{array}$ & $\begin{array}{c}47 \text { años } \\
(8.59)\end{array}$ & $\begin{array}{l}42 \text { años } \\
(11.94)\end{array}$ \\
\hline Mujeres & 6 & 45 & 12 & 6 & 69 \\
\hline $\begin{array}{c}\text { Edad } M \\
\text { M } \\
\text { (DT) }\end{array}$ & $\begin{array}{l}39 \text { años } \\
(13.57)\end{array}$ & $\begin{array}{c}36 \text { años } \\
(9.38)\end{array}$ & $\begin{array}{l}39 \text { años } \\
(10.61)\end{array}$ & $\begin{array}{l}43 \text { años } \\
(14.81)\end{array}$ & $\begin{array}{l}37 \text { años } \\
(10.52)\end{array}$ \\
\hline $\begin{array}{l}\text { Total de la } \\
\text { Categoría } \\
\text { Profesional }\end{array}$ & 23 & 114 & 29 & 16 & 182 \\
\hline $\begin{array}{c}\text { Edad Total } \\
\text { M } \\
\text { (DT) }\end{array}$ & $\begin{array}{l}44 \text { años } \\
(13.69)\end{array}$ & $\begin{array}{l}38 \text { años } \\
(10.91)\end{array}$ & $\begin{array}{l}44 \text { años } \\
(10.56)\end{array}$ & $\begin{array}{l}45 \text { años } \\
(10.95)\end{array}$ & $\begin{array}{l}40 \text { años } \\
(11.62)\end{array}$ \\
\hline Min.-máx. Edad & $27-73$ & $24-65$ & $30-71$ & $26-65$ & \\
\hline
\end{tabular}


Min.-máx. Edad27 - $7324-65 \quad 30-7126$ - 65 Nota 1. Asistentes engloba 1,2 y 3; Adjuntos abarcan 1, 2 y 3; Titular también incluye 1, 2 y 3 . La equivalencia aproximada de estas categorías de la Universidad de la Costa (Colombia) con las de las Universidades Públicas de España son: Catedrático $=$ Asociado; Asistente $=$ Becario Pre-doctoral $/$ Ayudante; Adjunto $=$ Ayudante $/$ Colaborador; Titular1 = Ayudante Doctor $/$ Contratado Doctor; Titular $2=$ Titular; Titular3 = Catedrático de Universidad.

En esta línea, al analizar la experiencia profesional previa en base a las categorías profesionales establecidas anteriormente (catedrático, asistente, adjunto y titular), se observan diferencias en cuanto a la efectuada en una institución y/o empresa externa y aquella vinculada al ámbito universitario (véase figura 1).

Figura 1. Experiencia profesional previa externa (empresa/institución ajena al ámbito universitario) y la Universitaria.

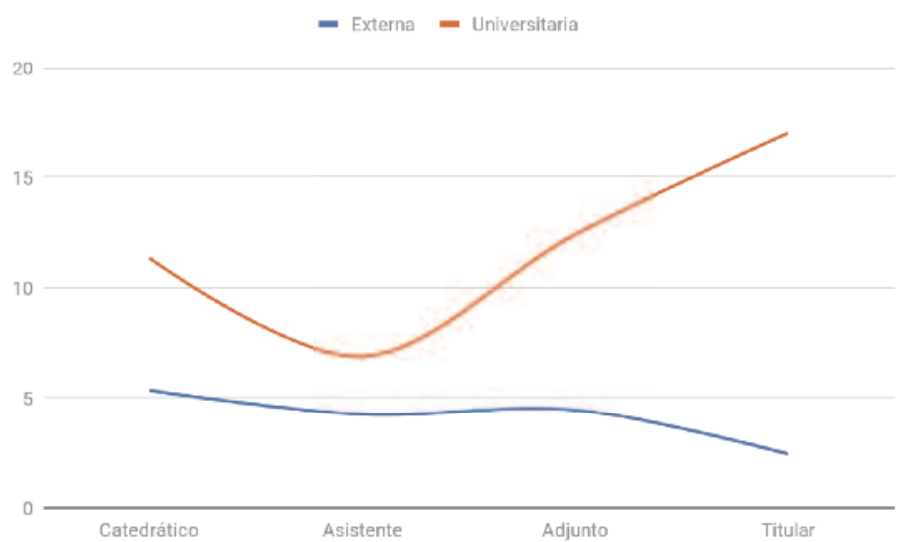

Asimismo, en relación a la comparativa entre los años de experiencia previa como docente universitario, se evidencia que el $0,71 \%(\mathrm{~N}=1)$ de los docentes afirman no tener, el 32,62\% $(\mathrm{N}=46)$, entre 1 y 3 años, el 20,57\% ( $N=29)$ entre 4 y 6 años, el 17,02\% ( $N=24)$ entre 7 y 12 años; el 9,2\% $(\mathrm{N}=13)$ entre 13 y 18 años; el $11,35 \%(\mathrm{~N}=16)$ entre 19 y 24 años y el $8,51 \%(\mathrm{~N}=12)$ de 25 en adelante (ver figura 2). 
Figura 2. Experiencia previa como docente universitario en base al género

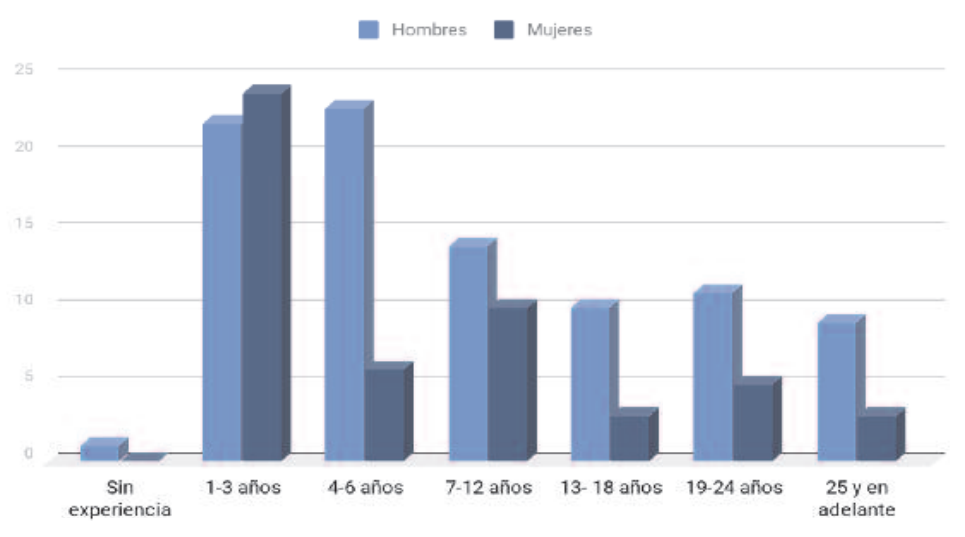

Igualmente, en cuanto a la compatibilización de la labor docente con otra actividad profesional, el $46,8 \%$ de los profesores encuestados no tienen, el 30,5\% desempeñan un ejercicio profesional libre y externo, el $12,8 \%$ trabajan en una empresa externa y el 9,9\% ejercen como docentes de enseñanza no universitaria.

\section{MÉTODO}

\section{Diseño}

Se realiza un estudio exploratorio que se materializa en la descripción, diseño y aplicación de un cuestionario ad hoc online, EMICOG, versión docente, que examina las metodologías educativas aplicadas por el profesorado de la Universidad de la Costa (Colombia) en distintas asignaturas de diversos planes de estudios. Este ha sido diseñado a través de la herramienta de la web 2.0, SurveyMonkey por su usabilidad, distribución aleatoria de ítems, exportación de los datos en distintos formatos y funciones avanzadas de análisis.

Instrumento de análisis sobre la evaluación académica, las metodologías docentes, las técnicas instruccionales y su incidencia en las Competencias Comunicativas Genéricas, EMICOG versión docente

El EMICOG - versión docente, abarca seis secciones de contenidos claramente diferenciadas: i) datos de carácter general, ii) técnicas y estrategias instruccionales, iii) recursos utilizados en la evaluación y enseñanza de la asignatura, iv) variables psicoeducativas, y v) necesidades formativas (ver Tabla 2)

\section{Datos de carácter general}

En la primera sección se presenta el estudio, se expone el propósito, la duración estimada y se solicita la colaboración de los docentes, haciendo mención en todo momento, a su carácter voluntario. En esta línea, y de acuerdo con el código deontológico, se afirma que los datos solamente se considerarán para análisis estadísticos globales sin interés individual y/o particular.

Con posterioridad, se pregunta a cada uno de los docentes si desean participar o no en el estudio (consentimiento informado) y a los que contestan afirmativamente, se les solicitan algunos 
datos demográficos tales como nombre, apellidos, número de identificación, fecha de nacimiento, edad, género, años de experiencia como profesor no universitario y como docente universitario, programa actual en el que imparte docencia, semestres, facultad, departamento, categoría laboral en la Universidad de la Costa, si está compatibilizando la actividad docente con alguna profesional, y en caso afirmativo, de qué tipo. Igualmente, se les pide indicar el nombre de la asignatura del semestre en la que consideran que han realizado un número mayor de actividades de enseñanza relacionadas con el uso de la construcción textual y/o lectura crítica, así como en el nombre de la que piensan que han realizado más actividades de evaluación relacionadas con dichas competencias genéricas. Y finalmente, que indiquen el nombre de la asignatura de referencia a la hora de contestar el instrumento.

Esta sección se completa con ocho ítems sobre el grado de conocimiento que tienen sobre las variables objeto de análisis y otros cuatro en torno al grado de interés para trabajar y/o evaluar la lectura crítica y la construcción textual en la asignatura seleccionada. Todo ello, mediante una escala tipo Likert de 5 puntos (ninguno, poco, ni poco ni mucho, bastante y mucho).

Tabla 2

Síntesis de las categorías del cuestionario ad hoc, EMICOG - versión docentes

\begin{tabular}{|c|c|c|c|}
\hline $\begin{array}{l}\text { Instrumento / } \\
\text { Constructo }\end{array}$ & Subprueba & Ítems & $\begin{array}{l}\text { Estudios previos de validación e } \\
\text { implementación }\end{array}$ \\
\hline \multicolumn{4}{|c|}{$D G$ - Datos generales } \\
\hline $\begin{array}{c}\text { Datos } \\
\text { demográficos }\end{array}$ & DG_DD & 20 & \multirow{2}{*}{$\begin{array}{l}\text { (Almerich, Orellana y Díaz-García, 2015; } \\
\text { Arias-Gómez et al., 2018; Barnet, 2001; } \\
\text { CUC, 2018; Díaz-Flórez, 2018; García- }\end{array}$} \\
\hline Conocimiento & DG-CON & 8 & \\
\hline Interés & DG-INT & 4 & $\begin{array}{c}\text { Martín y García-Sánchez, 2017; Gargallo- } \\
\text { López, Suárez-Rodríguez, Garfella-Esteban, } \\
\text { y Fernández, 2011; González, y Triviño, } \\
\text { 2018; OECD, 2016; Montanares, y Junod, } \\
\text { 2018) }\end{array}$ \\
\hline \multicolumn{4}{|c|}{ TE - Técnicas y estrategias instruccionales } \\
\hline \multirow{4}{*}{$\begin{array}{l}\text { Construcción } \\
\text { textual }\end{array}$} & $\begin{array}{l}\text { Estrategias y } \\
\text { técnicas } \\
\text { instruccionales }\end{array}$ & 6 & \multirow{4}{*}{$\begin{array}{l}\text { (De la Paz, Monte-Sano, Felton, Croninger, } \\
\text { Jackson y Worland Piantedosi, 2016; } \\
\text { Calderón, Parra y Piñeros, 2018; Graham, } \\
\text { 2018; Graham, Liu, Bartlett et al., 2018; } \\
\text { Graham, Liu, Ng et al., 2018; OECD, 2016) }\end{array}$} \\
\hline & $\begin{array}{l}\text { Géneros } \\
\text { textuales }\end{array}$ & 7 & \\
\hline & Procesos & 5 & \\
\hline & Medio & 4 & \\
\hline \multirow{3}{*}{ Lectura crítica } & $\begin{array}{l}\text { Estrategias y } \\
\text { Procesos }\end{array}$ & 6 & \multirow{3}{*}{$\begin{array}{c}\text { (Calderón, Parra y Piñeros, 2018; Halamish, } \\
\text { 2018; Graham, Liu, Bartlett et al., 2018; } \\
\text { Graham, Liu, Ng et al., 2018; OECD, } \\
\text { 2016;) }\end{array}$} \\
\hline & $\begin{array}{l}\text { Géneros } \\
\text { textuales }\end{array}$ & 7 & \\
\hline & Medio & 6 & \\
\hline
\end{tabular}




\begin{tabular}{|c|c|c|c|}
\hline \multicolumn{4}{|c|}{ EVEN - Evaluación y enseñanza de la asignatura } \\
\hline $\begin{array}{l}\text { Evaluación de } \\
\text { la asignatura }\end{array}$ & EV & 22 & $\begin{array}{l}\text { (Almerich, Orellana y Díaz-García, 2015; } \\
\text { CEEDAR, 2019a; 2019b; Gargallo-López } \\
\text { et al., 2011; Gonzales, 2018) }\end{array}$ \\
\hline $\begin{array}{l}\text { Enseñanza de } \\
\text { la asignatura }\end{array}$ & EN & 21 & $\begin{array}{l}\text { (Almerich, Orellana y Díaz-García, 2015; } \\
\text { Daumiller, Dickhäuser y Dresel 2019; } \\
\text { Gargallo-López et al., 2011; Gonzales, 2018; } \\
\text { González, y Triviño, 2018; Hatlevik y } \\
\text { Hatlevik, 2018; Montanares, y Junod, 2018; } \\
\text { Tesouro, Corominas, Teixidó, y Puiggalí, } \\
\text { 2014) }\end{array}$ \\
\hline \multicolumn{4}{|c|}{ PEP - Perfil Educativo y Psicológico } \\
\hline Autoeficacia & $\mathrm{AE}$ & 15 & $\begin{array}{l}\text { (Almerich, Orellana y Díaz-García, 2015; } \\
\text { García-Martín y García-Sánchez, 2017; } \\
\text { Gargallo-López et al., 2011; Gonzales, } \\
\text { 2013; Gonzales, 2018 ; Hatlevik y Hatlevik, } \\
\text { 2018; Lai, Hsiao y Hsieh, 2018; } \\
\text { Perandones, Herrera, y Lledó, 2013; } \\
\text { Pfitzner-Eden, 2016; Tesouro et al., 2014; } \\
\text { Zhao, y Zhang, 2017) }\end{array}$ \\
\hline $\begin{array}{l}\text { Dimensión } \\
\text { social }\end{array}$ & DS & 8 & $\begin{array}{c}\text { (Daumiller, Dickhäuser y Dresel 2019; } \\
\text { García-Martín y García-Sánchez, 2017; } \\
\text { Gonzales, 2013; Gonzales, 2018; Zhao, y } \\
\text { Zhang, 2017) }\end{array}$ \\
\hline $\begin{array}{l}\text { Inteligencia } \\
\text { emocional }\end{array}$ & PEP_IE & 7 & $\begin{array}{l}\text { (Daumiller, Dickhäuser y Dresel 2019; } \\
\text { García-Martín y García-Sánchez, 2017; } \\
\text { Gonzales, 2013; Perandones, Herrera, y } \\
\text { Lledó, 2013; Pfitzner-Eden, 2016; Torrijos, } \\
\text { Martín, y Rodríguez, 2018) }\end{array}$ \\
\hline Motivación & $\underset{\text { TOT }}{\text { MOT_ATR }}+$ & 6 & $\begin{array}{l}\text { (Daumiller, Dickhäuser y Dresel 2019; } \\
\text { Gargallo-López et al., 2011; Lai, Hsiao y } \\
\text { Hsieh, 2018; Montanares, y Junod, 2018; } \\
\text { Torrijos, et al., 2018; Zhao, y Zhang, 2017) }\end{array}$ \\
\hline Atribuciones & & 2 & $\begin{array}{l}\text { (Daumiller, Dickhäuser y Dresel 2019; } \\
\text { García-Martín y García-Sánchez, 2017) }\end{array}$ \\
\hline Afrontamiento & AFR & 13 & $\begin{array}{l}\text { (Daumiller, Dickhäuser y Dresel 2019; } \\
\text { García-Martín y García-Sánchez, 2017) }\end{array}$ \\
\hline
\end{tabular}




\begin{tabular}{|c|c|c|}
\hline \multicolumn{3}{|c|}{ NNFF - Necesidades formativas } \\
\hline Aprendizaje & 4 & $\begin{array}{l}\text { (Daumiller, Dickhäuser y Dresel 2019; } \\
\text { García-Martín y García-Sánchez, 2017; } \\
\text { Gargallo-López et al., 2011; Gonzales, } \\
\text { 2013; Gonzales, 2018; González, y Triviño, } \\
\text { 2018; Halamish, 2018; Torrijos, et al., } \\
\text { 2018) }\end{array}$ \\
\hline $\begin{array}{c}\text { Herramientas } \\
\text { web }\end{array}$ & 10 & $\begin{array}{c}\text { (Almerich, Orellana y Díaz-García, 2015; } \\
\text { García-Martín y García-Sánchez, 2017; } \\
\text { García-Martín, Merchant y García-Sánchez, } \\
\text { 2016; Gargallo-López et al., 2011; } \\
\text { González, y Triviño, 2018; Hatlevik y } \\
\text { Hatlevik, 2018;) }\end{array}$ \\
\hline
\end{tabular}

\section{Técnicas y estrategias instruccionales}

En la segunda sección, se solicita a los docentes que indiquen si alguna vez, han pedido realizar en sus asignaturas, tareas como la elaboración de resúmenes y/o esquemas, la toma de apuntes, la construcción de mapas conceptuales, la realización de anotaciones, de auto-preguntas, acrósticos... para completar la información, para recordarla y/o para guiar los procesos de construcción textual tanto en el momento de planificación como en el de revisión. Todo ello, en base a una escala tipo Likert de 5 puntos (nunca, casi nunca, a veces, casi siempre y siempre).

Igualmente, se les pide indicar si han utilizado en la asignatura a examen, distintas tipologías textuales tales como los textos argumentativos, los de comparación y contraste, los de causa-efecto y/o problema-solución, los ensayos, las reseñas bibliográficas, las revisiones sobre el estado de la cuestión, tanto a la hora de enseñar/trabajar un tema como a la de evaluar el aprendizaje, en base a una escala tipo Likert de 5 puntos (nunca, casi nunca, a veces, casi siempre y siempre). Al mismo tiempo que concretan sus percepciones sobre la utilidad de dichas tipologías en la asignatura en análisis, en base a otra escala tipo Likert de 5 puntos (muy baja, baja, ni baja ni alta, alta y muy alta).

Del mismo modo, se les insta en base a una escala tipo Likert de 5 puntos (nunca, casi nunca, a veces, casi siempre y siempre) sobre los procesos psicológicos superiores implicados en cualquier tarea de composición escrita (planificación, edición y revisión) con independencia de la tipología textual, así como sobre los medios utilizados en la implementación de las estrategias de enseñanza.

\section{Recursos utilizados en la evaluación y enseñanza de la asignatura}

En la tercera sección, se les solicita especificar en qué medida, siguiendo una escala tipo Likert de 5 puntos (nunca, casi nunca, a veces, casi siempre y siempre), utilizan los diversos recursos mencionados para evaluar la asignatura objeto de estudio. Entre estos, se examinan los exámenes escritos de preguntas cortas, los de ensayo, las pruebas objetivas escritas, los análisis de trabajos escritos, la participación en debates, los análisis de presentaciones, los escritos de aplicaciones prácticas, los análisis escritos de lecturas de casos prácticos, de publicaciones científicas, la autoevaluación del aprendizaje, la coevaluación..., la elaboración y contribución en wikis y blogs que abordan algún problema nuclear de la asignatura... Esta escala se completa con una pregunta abierta en la que se les solicita que expliquen brevemente el sistema de evaluación de la asignatura. 
A continuación, se les pide que determinen en qué medida utilizan los distintos recursos (las lecciones magistrales, las charlas y conferencias, las lecturas y elaboración de textos argumentativos, las actividades de trabajo de campo, los casos prácticos, los proyectos, los debates, las dinámicas grupales, la solución de problemas, las clases con ordenador y herramientas virtuales en la nube...), durante el proceso de enseñanza de los contenidos de la asignatura examinada. Igualmente, esta escala se complementa con una pregunta abierta en la que se les solicita que describan brevemente la metodología docente empleada en la asignatura.

\section{Variables psicoeducativas}

En la cuarta, se examinan las percepciones y creencias que los docentes encuestados tienen en torno a diferentes variables psicoeducativas tales como autoeficacia, inteligencia emocional, motivación, atribuciones, dimensión social y estrategias de afrontamiento, en base a escalas tipo Likert de 507 puntos (ver tabla 3).

Tabla 3

Síntesis de las variables psicoeducativas del cuestionario, EMICOG - versión docente

\begin{tabular}{|c|c|c|c|}
\hline & $\begin{array}{l}\mathbf{N}^{0} \text { de } \\
\text { ítems }\end{array}$ & Ejemplo de ítem & Escala \\
\hline Autoeficacia & 15 & $\begin{array}{l}\text { ¿En qué medida se siente capaz, en su asignatura, } \\
\text { para sus estudiantes, de... enseñar a aprender de } \\
\text { forma autónoma? }\end{array}$ & Likert 7 \\
\hline $\begin{array}{l}\text { Inteligencia } \\
\text { Emocional }\end{array}$ & 7 & $\begin{array}{l}\text { ¿Con qué frecuencia expresa sus sentimientos } \\
\text { abiertamente? }\end{array}$ & Likert 7 \\
\hline Motivación & 3 & $\begin{array}{l}\text { ¿Hace las cosas para obtener reconocimiento y/o } \\
\text { aprobación de los demás? }\end{array}$ & Likert 7 \\
\hline Atribuciones & 2 & $\begin{array}{l}\text { Cuando no consigue alcanzar sus metas, } \\
\text { habitualmente piensa que se } \text { debe } \\
\text { esfuerzo...capacidad... buena suerte... }\end{array}$ & Likert 5 \\
\hline $\begin{array}{l}\text { Dimensión } \\
\text { social }\end{array}$ & 8 & $\begin{array}{l}\text { Puede contar con personas que le reconfortan y le } \\
\text { animan }\end{array}$ & Likert 7 \\
\hline Afrontamiento & 13 & $\begin{array}{l}\text { Cuando se encuentra ante alguna dificultad o } \\
\text { problema, ¿se niega a creer lo que está pasando y } \\
\text { trata de engañarse a sí mismo/a? }\end{array}$ & Likert 7 \\
\hline
\end{tabular}

\section{Necesidades formativas}

En la quinta y última sección, se requiere a los profesores encuestados que indiquen su grado de interés y de necesidad en recibir formación sobre la enseñanza y evaluación de textos académicos tales como Trabajos Fin de Grado (TFG) y/o Trabajos Fin de Máster (TFM); las herramientas web, Ios M00Cs, los sistemas de almacenamiento en la nube (Google Drive)..., los entornos virtuales de aprendizaje, el diseño de encuestas, la consulta de bases de datos, los distintos estilos de citación bibliográfica, la organización del tiempo... atendiendo a una escala tipo Likert de 5 puntos (nada necesario, poco necesario, ni poco ni mucho, necesario y muy necesario). 


\section{SISTEMAS DE EVALUACIÓN Y METODOLOGÍAS DOCENTES Y SU INCIDENCIA EN LAS COMPETENCIAS GENÉRICAS (EMICOG - PROFESORES)}

\section{DISCUSIÓN Y CONCLUSIONES}

La aplicación del cuestionario online, EMICOG - versión docentes, al profesorado de la Universidad de la Costa (Colombia) permite, por una parte, determinar cuáles son las metodologías docentes utilizadas con mayor frecuencia y las que menos, y por otra, identificar las posibles razones o motivos de dicha preferencia. Del mismo modo, favorece la delimitación de las necesidades formativas del profesorado encuestado, que serán la base de futuros programas/cursos instruccionales de estrategias eficientes para la mejora de la enseñanza, repercutiendo en la consecución de mayores logros académicos.

Igualmente, el examen de las variables psicoeducativas como la autoeficacia, la motivación, la inteligencia emocional, las estrategias de afrontamiento... (García-Martín y García-Sánchez, 2017), es decisivo a la hora de determinar el enfoque de enseñanza a seguir, las estrategias a implementar de forma transversal, así como los géneros textuales y medios de uso predominante, mejorando en último término la calidad docente.

Por todo ello, sería conveniente que se realizasen investigaciones internacionales futuras con el fin de comparar el uso de dichas metodologías docentes en diversos países en vistas a poder delimitar patrones de uso diferenciales y por consiguiente estrategias instruccionales eficaces y eficientes en función del área geográfica de desarrollo.

\section{REFERENCIAS BIBLIOGRÁFICAS}

Almerich, G., Orellana, N., y Díaz-García, I. (2015). Las competencias en TIC en el profesorado en formación y su relación con las creencias pedagógicas, la autoeficacia y la percepción de impacto de las TIC en la educación. En AIDIPE (Ed.), Investigar con y para la sociedad (Vol. 2, pp. 589597). Cádiz, España: Bubok. Recuperado de http://aidipe2015.aidipe.org

Arias, D. H., Díaz, O. C., Garzón, I., León, A. C., Rodríguez, S. P., y Valbuena, E. O. (2018). Entre las exigencias de calidad y las condiciones de desigualdad: Formación inicial de profesores en Colombia. Bogotá: UPN.

Barnet, R. (2001). Los límites de la competencia. El conocimiento, la educación superior y la sociedad. Barcelona: Gedisa.

Calderón, A., Parra, C. A., y Piñeros, M. A. (2018). Guía de orientación Saber Pro: Módulos de competencias genéricas. Bogotá, Colombia: Instituto Colombiano para la Evaluación de la Educación (Icfes).

CUC. (2018). Autoinforme de Acreditación. Barranquilla: CUC

Daumiller, M., Dickhäuser, 0., y Dresel, M. (2019). University instructors' achievement goals for teaching. Journal of Educational Psychology, 111(1), 131-148. doi: 10.1037/edu0000271

De la Paz, S., Monte-Sano, C., Felton, M., Croninger, R., Jackson, C., y Worland Piantedosi, K. (2016). A historical writing apprenticeship for adolescents: integrating disciplinary learning with cognitive strategies. Reading Research Quarterly, 52(1), 31-52. doi: 10.1002/rrq.147

Díaz-Flórez, 0. C. (2018). Las competencias en la educación superior. Debates contemporáneos. Tesis Doctoral. Bogotá: UPN

García-Martín J., y García-Martín, S. (2018). Los M00CS: Estado de la cuestión y propuesta educativa. En E. López-Meneses, D. Cobos Sanchiz, A. H. Martín Padilla, L. Molina García y A. Jaén Martínez (Eds.). Experiencias pedagógicas e innovación educativa: Aportaciones desde la praxis docente e investigadora (pp. 2935-2942). Barcelona: Octaedro.

García-Martín, J., Merchant, G., y García-Sánchez, J. N. (2016). Preparing to teach 21 st Century literacies. En C. Kosnik, S. White, C. Beck, B. Marshall, A. L. Goodwin y J. Murray (Eds.). Building Bridges. Rethinking Literacy Teacher Education in a Digital Era (pp. 43 - 55). Rotterdam: Sense Publishers 
García-Martín, J., y García-Sánchez, J. N. (2018). The instructional effectiveness of two virtual approaches: processes and product. Journal of Psychodidactics, 23(2), 117-127. doi: 10.1016/j.psicod.2018.02.002

García-Martín, J., y García-Sánchez, J. N. (2013). Patterns of web 2.0 tool use among young Spanish people. Computers and Education, 67(3), 105-120. doi: 10.1016/j.compedu.2013.03.003

García-Martín, J., y García-Sánchez, J. N. (2015). Use of Facebook, Tuenti, Twitter and Myspace among young Spanish people. Behaviour \& Information Technology, 34(7), 685-703. doi: 10.1080/0144929X.2014.993428

García-Martín, J., y García-Sánchez, J. N. (2017). Pre-service teachers' perceptions of the competence dimensions of digital literacy and of psychological and educational measures. Computers \& Education, 107, 54-67. doi: 10.1016/j.compedu.2016.12.010

García-Martín, J., y García-Sánchez, J. N. (2019). Promoción de las competencias para la vida a través de la gamificación en un M00C. En D. Padilla Góngora, J. M. Aguilar Parra y R. López Liria (Eds.). Salud y ciclo vital (pp. 289-296). Madrid: Editorial DYKINSON.

Gargallo-López, B., Suárez-Rodríguez, J., Garfella-Esteban, P. R., y Fernández-March, A. (2011). El cuestionario CEMEDEPU. Un instrumento para la evaluación de la metodología docente y evaluativa de los profesores universitarios. Estudios sobre Educación, 21, pp. 9-40.

Gonzales, M. C. (2013). Influencia de la autoeficacia docente colectiva en el profesorado universitario. Psicología Educativa, 3-12. doi: 10.5093/ed2013a2

Gonzales, M. C. (2018). Construcción de la escala de autoeficacia docente colectiva del profesorado universitario. Revista Espacios, 39(52), 30-38.

González, S. y Triviño, M. A. (2018). Las estrategias didácticas en la práctica docente universitaria. Profesorado. Revista de curriculum y formación del profesorado, 22(2), 371-388. doi: 10.30827/profesorado.v22i2.7728

Graham, S. (2018). A revised writer(s)-within-community model of writing. Educational Psychologist, 53(4), 258-279. doi: 10.1080/00461520.2018.1481406

Graham, S., Liu, X., Bartlett, B. Ng, C., Harris, K. R., Aitken, A., Barkel, A., Kavanauge, C., y Talukdar, J. (2018). Reading for writing: a meta-analysis of the impact of reading interventions on writing. Review of Educational Research, 88(2), 243-284. doi: 10.3102/0034654317746927

Graham, S., Liu, X., Ng, C., Bartlett, B., Harris, K. R., y Kavanauge, C. (2018). Effectiveness of literacy programs balancing reading and writing instruction: a meta-analysis. Reading Research Quarterly, 53(3), 279-304. doi: 10.1002/rrq.194

Halamish, V. (2018) Pre-service and In-service teachers' metacognitive knowledge of learning dtrategies. Frontiers in Psychology, 9(2152), pp. 1-5. doi: 10.3389/fpsyg.20 18.02152

Hatlevik, I. K. R., y Hatlevik, O. E. (2018). Examining the relationship between teachers' ICT self-efficacy for educational purposes, collegia collaboration, lack of facilitation and the use of ICT in teaching practice. Frontiers in Psychology, 9(935), pp. 1-8. doi: 10.3389/fpsyg.2018.00935

Hew, K. F., y Cheung W. S. (2014). Students' and instructors' use of massive open online courses (M00Cs): Motivations and challenges. Educational Research Review, 12, 45-58. doi: 10.1016/j.edurev.2014.05.001

Lai, H.-M., Hsiao, Y-L., y Hsieh, P-J. (2018). The role of motivation, ability, and opportunity in university teachers' continuance use intention for flipped teaching. Computers \& Education, 124, 37-50. doi: 10.1016/j.compedu.2018.05.013

Liaw, S. S., y Huang, H. S. (2013). Perceived satisfaction, perceived usefulness and interactive learning environments as predictors to self-regulation in e-learning environments. Computers \& Education, 60, 14-24. doi: 10.1016/j. compedu.2012.07. 01 
Lin, W. S. (2012). Perceived fit and satisfaction on web learning performance: IS continuance intention and task-technology fit perspectives. International Journal of Human-Computer Studies, 70, 498-507. doi: 10.1016/j.jijhcs.2012 .01.006

Montanares, E. G., y Junod, P. A. (2018). Creencias y prácticas de enseñanza de profesores universitarios en Chile. Revista Electrónica de Investigación Educativa, 20(1), 93-103. doi: 10.24320/redie.2018.20.1.1383

OECD. (2016). La educación en Colombia. MEC: OECD. Recuperado de: https://www.mineducacion.gov.co/1759/articles-356787 recurso 1.pdf

Perandones, T. M., Herrera, L., y Lledó, A. (2013). Felicidad subjetiva y autoeficacia docente en profesorado de República Dominicana y España. European Journal of Investigation in Health, Psychology and Education, 3(3), 277-288. doi: 10.1989/ejihpe.v3i3.50

Pfitzner-Eden, F. (2016). Why Do I Feel More Confident? Bandura's Sources Predict Preservice Teachers' Latent Changes in Teacher Self-Efficacy. Frontiers in Psychology, 7(1486), pp. 1-16. doi: 10.3389/fpsyg.2016.01486

Tesouro, M., Corominas, E., Teixidó, J., y Puiggalí, J. (2014). La autoeficacia docente e investigadora del profesorado universitario: relación con su estilo docente e influencia en sus concepciones sobre el nexo docencia-investigación. Revista de Investigación Educativa, 32 (1), 169-186. doi: 10.6018/rie.32.1.172771

Torrijos, P., Martín, J. F., y Rodríguez, M. J. (2018). La educación emocional en la formación permanente del profesorado no universitario. Profesorado. Revista de currículum y formación del profesorado, 22(1), 579-597.

Zhao, H., y Zhang, X. (2017). The influence of field teaching practice on Pre-service Teachers' Professional Identity: A Mixed Methods Study. Frontiers in Psychology, 8(1264), 1-11. doi: 10.3389/fpsyg.2017.01264 